\title{
BM Global Health Responding to ever-changing epidemiological dynamics of Ebola virus disease
}

\author{
Yuki Maehira, ${ }^{1}$ Yohei Kurosaki, ${ }^{2}$ Tomoya Saito, ${ }^{3}$ Jiro Yasuda, ${ }^{2}$ Masayoshi Tarui, ${ }^{4}$ \\ Denis J M Malvy, ${ }^{5}$ Tsutomu Takeuchi ${ }^{1}$
}

To cite: Maehira $Y$, Kurosaki Y, Saito T, et al. Responding to ever-changing epidemiological dynamics of Ebola virus disease. BMJ Global Health 2016;1: e000180. doi:10.1136/ bmjgh-2016-000180

Received 6 September 2016 Accepted 27 October 2016

\section{CrossMark}

\footnotetext{
${ }^{1}$ St. Luke's International University, Tokyo, Japan ${ }^{2}$ Institute of Tropical Medicine (NEKKEN), Nagasaki University, Nagasaki, Japan

${ }^{3}$ National Institute of Public Health, Saitama, Japan

${ }^{4}$ Keio University, Tokyo, Japan

${ }^{5}$ Inserm 1219, University of Bordeaux \& Division of Clinical Tropical Medicine, CHU de Bordeaux, Bordeaux, France
}

\section{Correspondence to}

Yuki Maehira;

maehiray@snow.ocn.ne.jp

\section{ABSTRACT}

With the incidence and mortality rates of Ebola virus disease (EVD) in Guinea, Liberia and Sierra Leone now at zero and reports of the largest and most complex EVD outbreak in history no longer on the front pages of newspapers worldwide, the urgency of that crisis seems to have subsided. During this lull after the storm and before the next one, the international community needs to engage in a 'lessons-learned' exercise with respect to our collective scientific, clinical and public health preparedness. This engagement must identify pragmatic, innovative mechanisms at multinational, national and community levels that allow research and development of next generation diagnostics and therapeutics, the safe and effective practice of medicine, and the maintenance of public health to keep pace with the rapid epidemiological dynamics of EVD and other deadly infectious diseases.

\section{SHORTENING THE DISEASE TRANSMISSION CHAIN}

The 2014-2015 Ebola virus disease (EVD) outbreak was exacerbated by poorly operating or non-existent healthcare facilities and the stigma attached to afflicted individuals who journeyed to those facilities that were in operation. Simulations based on data from an observational study in Guinea of EVD transmission from February to August 2014 showed 'that a $10 \%$ increase in hospital admissions could have reduced the length of (transmission) chains by $26 \%$ '. The point is that, in future EVD epidemics, individuals who are either symptomatic or at risk must be encouraged to visit care facilities. This requires strengthening of persistently weak community healthcare systems and converting the view of treatment centres by patients, their families and their communities not as places at which to die, but at which to be cured.

In many regions where well-integrated healthcare infrastructures have been slow to develop or have been destroyed by years of

\section{Key questions}

What is already known about this topic?

- While the global medical research and development (R\&D) race was intensively performed, the much needed medical countermeasures have not yet materialised to diagnose and treat Ebola virus disease (EVD).

- The alert of WHO PHEIC had been lifted in the end of March 2015 and global R\&D interest is going on to other things such as Zika virus infection, however, each health system in the affected countries has not sufficiently been restored for future response to new EVD cases.

\section{What are the new findings?}

- There are potential products against EVD; however, those should further be validated to ensure the efficacy, usability from the patientcentred, community-focused viewpoints.

- The clinical trial conducted in Guinea (JIKI study) has proven the feasibility of conducting clinical research in the midst of an epidemic where research literacy is far from enough for a continuous involvement in preparation and implementation at field level.

- Involving industries, translational collaboration can offer new insights to produce and deploy the life-saving solutions, developing cultural sensitivity to build trust within communities and flexibility to adapt clinical research to field constraints.

\section{Recommendations for policy}

- R\&D and clinical case management strategies should be strategically integrated with social mobilisation measures to establish a supportive local environment for public health outcomes.

- A strategic multilayered intervention can fulfil the gap in short-term activity such as the vaccination programme in high-risk level contacts to EVD to provide more solid measures for prevention and healthcare benefit to vulnerable populations and societies.

- Innovative governance systems should be established along with a professional portfolio management mechanism for responses to everchanging epidemiological dynamics of EVD at both international and national levels. 
war and violence, it is not practical to expect people at risk for EVD to voluntarily come to urban-centred emergency care centres. In the case of the 2014-2015 EVD epidemic in West Africa, these Ebola Treatment Units ('ETUs') were too physically, culturally and psychologically distant from the local communities where the outbreak was rampant.

Physical distance: The physical distance between patient and treatment-which lengthens the transmission chain -arose from the long distances between local communities and the urban-based ETUs, rendered even longer by the absence of an operating transportation infrastructure. The nature of EVD exacerbated this distance because the resources for confirmatory diagnosis of the disease were centralised at the ETUs. The capacity to more immediately and precisely diagnose symptomatic patients and individuals at risk due to exposure at decentralised points of care could dramatically contribute to controlling threats of disease expansion with less complexity and use of fewer resources. Establishing alternative channels for timely diagnosis and access to care will also contribute to more effective and efficient enrolment in clinical studies of experimental treatments and reduce probable selection biases.

Cultural distance: The cultural distance between patient and diagnosis and treatment arose from the palpably foreign nature of the healthcare services and technologies at the ETUs. In this context, patients were prone to feel not like patients but like refugees outcast from their families and communities. This problem can be addressed in significant part through long-term intensive communications programmes in local languages and dialects that promote and strengthen awareness of the causes of EVD and the methods of diagnosing and treating it.

Psychological distance: The physical and cultural distances combined to create a psychological distance between patient and diagnosis and treatment, with the death spiral effect of all three evidenced by the evergrowing incidence of EVD and associated mortality.

\section{SHORTENING THE TIME TO TREATMENT}

Among the clinical trials ${ }^{2}{ }^{3}$ of experimental drugs for EVD, the pioneer study led by the French Institut National de la Santé et de la Recherche Médicale (Inserm $)^{45}$ found that on average it took up to 5 days for treatment to begin after confirmed diagnosis at an ETU. The time to travel from the local community to an ETU meant that the time between onset of symptoms or exposure and treatment was even longer. These delays in diagnosis and treatment were in stark conflict with the need to administer antiviral therapeutics as soon as possible.

In addition to the delay to diagnose and treat caused by distance, two additional sources of delay arise in this context: first, negative diagnoses resulting from a lower viral load before symptoms appear; second, missed opportunities to treat asymptomatic individuals who have had contact with confirmed infected individuals and who could have benefited from timely postexposure prophylactic (PEP) treatment.

\section{CLINICAL STUDIES OF EXPERIMENTAL DRUGS TO TREAT EVD}

All of these issues add complexity to the quest to obtain quantitatively meaningful data from clinical studies of experimental drugs to treat EVD. Moreover, this quest competes against the local health outcome-based case management objective to treat individual patients and control the spread of the disease. This conflict becomes particularly significant in the case of treatment with experimental drugs where there may not be sufficient time to reduce the risk that adverse effects that may have been observed in non-human primate studies will carry over into humans. ${ }^{6}$ Using experimental drugs in patients who have a negative diagnosis (even if falsely negative) or where there is an absence of symptoms presents further ethical issues.

The WHO now requires phase 3 level incentive surveillance in the three affected countries ${ }^{7}$ and some flare-up cases have been detected, assuming sexual transmission because of persistent residual virus in the sperm of recovered men. ${ }^{8-10}$ Case investigation efforts now require vigorous postmortem screening as part of safe burial promotions for active mobilisation of communities. Simultaneously, the driving forces of control measures have been changed from foreign health emergency response teams and R\&D experts to local health government agencies. The landscape in any affected county is always changing and it has become difficult for local authorities to identify the best public health countermeasures adapted to each local context because of a multitude of social uncertainties (figure 1).

Japan's historic role in responding to global health emergencies is often viewed as simply that of a financial supporter, with no physical presence. In the case of the 2014-2015 EVD outbreak, however, Japan actively sought to provide medical $\mathrm{R} \& \mathrm{D}$ resources in collaboration with and the support of various international partners. We offer here two examples of this with respect to the EVD epidemic in Guinea: a field test of a rapid EVD test kit using the Reverse Transcription Loop-mediated Isothermal Amplification (RT-LAMP) method ${ }^{11}$ and a clinical study of the small-molecule drug favipiravir. ${ }^{12} 13$

\section{The benefits of the RT-LAMP method as a community-based diagnostic}

In discussing the value of any diagnostic test for EVD and similar haemorrhagic virus infections, we first note the extreme danger of false-negative results. To be effective at a local village level, EVD diagnostics must have high negative predictive value along with specificity and sensitivity to detect EVD, without cross-reactivity to other haemorrhagic fever viruses and arboviruses (which 


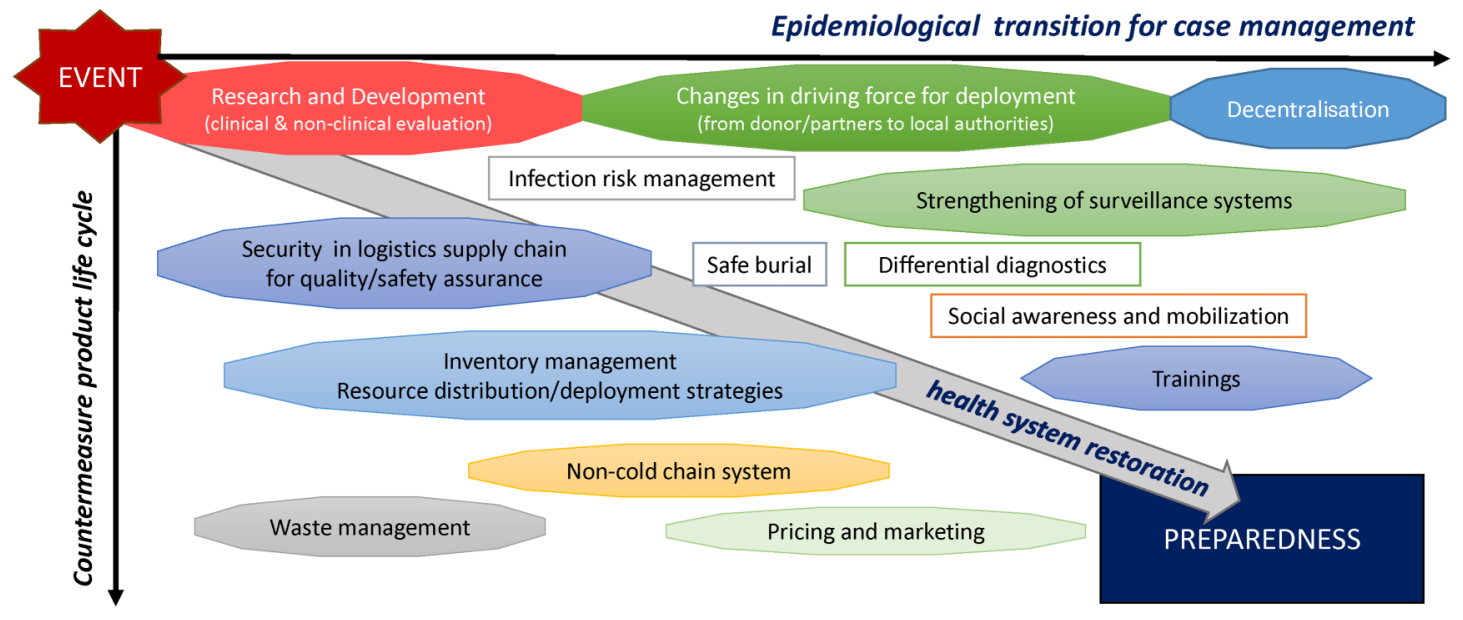

Figure 1 Adjusting to ever-changing dynamics for deployment of medical countermeasures.

cause similar acute febrile diseases). We believe that the RT-LAMP diagnostic tested in Guinea during the 20142015 EVD outbreak may satisfy these requirements more so than the fingerstick-based rapid diagnostic testing (RDT) immunoassay tool that was widely deployed during the outbreak. ${ }^{14} 15$

Some argue that the RDT system itself cannot be used as the sole basis for patient management ${ }^{14}$ due to its limitations as to sensitivity and specificity in comparison with the real-time Reverse-Transcription PCR system (rRT-PCR).$^{16}$ Compared to the danger of a missed detection of malaria due to a false-negative result, there is much greater danger in missing a patient with EVD due to a false negative; thus, RDT-positive cases must be transferred to the ETUs for confirmatory diagnosis. Notwithstanding these limitations, proponents of the RDT test argue that it was used efficiently for primary diagnostics to warn of infection risk for those deployed to conduct community outreach investigations. These proponents note that the ReEBOV Antigen Rapid Test (Corgenix, USA) showed preferably high sensitivity and specificity in its field validation results ${ }^{17} 18$ and that the fingerstick used in this test had undergone some improvement since its factory-based validation and WHO evaluation study. ${ }^{19}$ Additionally, the RDT, as an immunoassay, requires a $10^{3}{ }^{4}$ times higher cycle threshold (Ct) value than rRT-PCR for detection, while the lower limit of detection of PCR varies based on the procedures. $^{20} 21$

RDT proponents argue that rRT-PCR is not completely reliable despite being regarded as the WHO benchmark reference system. ${ }^{22} 23$ They also point to the high acquisition costs of rRT-PCR platforms and the ongoing expense of technical expertise for routine deployment in the health systems of developing countries where the priorities are misplaced and the funds misspent. With this criticism in mind, we note, however, that, aiming for decentralised point-of-care testing with remote monitoring in a wide variety of laboratory settings, a fully automated RT-PCR system, Xpert Assay (Cepheid, USA), has been evaluated and can be performed within 90-100 min from sample acquisition. ${ }^{2425}$

Given this debate over diagnostic methods, it is clear that further development is needed in the scientific advancement of diagnostic technology to ensure high negative predictive value for EVD cases and greater robustness to detect the presence of virus in sample specimens the integrity of which is subject to the challenges posed by environments such as those found in West Africa during the 2014-2015 EVD outbreak. This also suggests the necessity of developing new screening algorithms and adapting to a continuum of EVD care to account for the detection of infected individuals presenting with lower viraemia-both symptomatic and asymptomatic patients.

The RT-LAMP method developed through an academic-industry collaboration in Japan was validated in Guinea in March 2015 and showed significantly higher sensitivity concordant with the results from rRT-PCR, particularly with the primers for the trailer region of the Ebola virus genome $(100 \%$ for all indicators of sensitivity, specificity, negative and positive predictive values). ${ }^{26}$ The RT-LAMP test kit also showed applicability to oral swabs (in contrast to blood samples) for higher throughput screening. Table 1 presents data that compare RT-LAMP with the RDT and rRT-PCR. By validating the use of RT-LAMP in Guinea's tropical climate, these data suggest that the RT-LAMP method has the potential to be deployed for rapid and accurate point-of-care diagnosis in future EVD outbreaks in regions where the Ebola is endemic.

Owing to the data presented in table 1, the RT-LAMP test kit (in the portable platform of Genie III, Toshiba Medical Systems Corp., Japan) has been well received, particularly by local health policymakers and laboratory technicians in Guinea, as official bilateral aid. With expectations that its simple, practical usability in field settings will support point-of-care diagnostics for individual case management, the RT-LAMP test kit can be operated by battery. The RT-LAMP test kit has also been 
Table 1 Comparison of EVD diagnostic systems

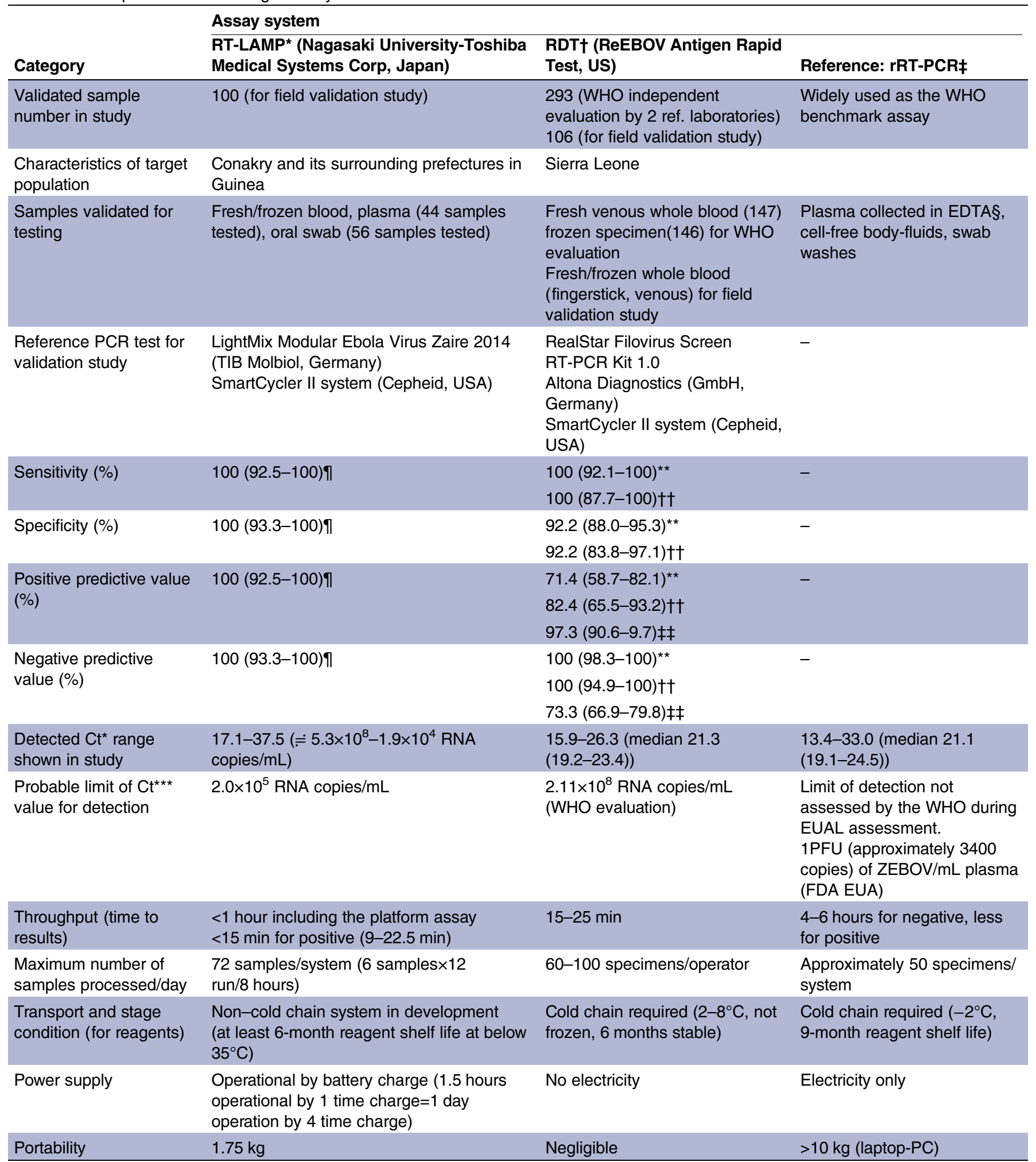

${ }^{*}$ RT-LAMP, reverse transcription-loop mediated amplification method.

†RDT, rapid diagnostic test.

¥RRT-PCR, real-time reverse transcription-PCR.

§EDTA, ethylenediamine-tetraacetic acid.

qThe indicated results were from the RT-LAMP test with the primers for the untranscribed trailer region with 100 samples. $^{26}$

${ }^{\star \star}$ The indicated results were obtained by the WHO evaluation test at the reference laboratory with 277 samples (whole blood). ${ }^{17}$

††The indicated values were obtained by the field validation study with 105 fingerstick samples. ${ }^{17}$

$\ddagger \ddagger T$ The indicated values were obtained by the field validation study with 176 samples (whole blood). ${ }^{18}$

${ }_{* \star \star} \mathrm{Ct}$, cycle threshold.

EVD, Ebola virus disease; EUA, emergency use authorisation; FDA, Food and Drug Administration. 
used with timely sampling with oral swabs as a postmortem diagnostic to purposively support safe burial in communities in Guinea. More than 2000 postmortem cases were tested between April 2015 and August 2016 (unpublished data). If rRT-PCR is not available for confirmatory diagnosis of EVD, RT-LAMP can be alternatively deployed for efficient decision-making and reduction of the health resource burden. ${ }^{27}$

Recently, RT-LAMP in combination with a new extraction kit, SpeedXtract (Qiagen, Germany), and an improved diagnostic reverse transcription recombinase polymerase amplification assay (EBOV-RT-RPA), has been validated on sera and swabs in Guinea for case management as well as for safe burial management. ${ }^{28}$ The result on this combined device showed higher sensitivity than the RealStar Filovirus rRT-PCR kit (Altona-Diagnostics, Germany), and provided significantly fast results, within 30-60 $\mathrm{min}$, with comparatively high specificity and sensitivity to the results shown by the above RT-LAMP method. ${ }^{26}$

A point-of-care diagnostic system such as RT-LAMP is essential to precisely screen and confirm each infection risk and case management in local settings. Moreover, when combined with PEP therapy discussed in more detail below, RT-LAMP can support treatment-forprevention measures and follow-up intervention for those at risk of sexual transmission.

\section{THE POTENTIAL FOR FAVIPIRAVIR AS A MEDICAL COUNTERMEASURE AGAINST THE EBOLA VIRUS}

The 2014-2015 EVD outbreak spurred efforts to develop a vaccine against the Ebola virus. To this end, the results of efforts such as the rVSV-EBOV ring vaccination clustered-randomised trial offer some hope. ${ }^{29}$ However, even if these vaccination efforts succeed, there may still be a 10-day delayed onset of immunity after vaccination, ${ }^{30}{ }^{31}$ and in diseases like EVD, there is a 2-day to 20-day incubation period, ${ }^{32}$ with higher levels of exposure during the advanced excretion stage of the disease. A PEP, therefore, could be an important tool for minimising the impact and spread of infection before a vaccine would work in any risk person. We believe that the small molecule drug favipiravir (developed by Toyama Chemical Co, a company within FUJIFILM Corp, Japan) has the potential to be used for this purpose.

Favipiravir was the drug tested in the JIKI clinical study in Guinea, which was supported by the Japanese government and undertaken in collaboration with the government of Guinea, Inserm, Médecins Sans Frontières, the Alliance for International Medical Action and Toyama Co. The results of the JIKI study did not allow for any statistically significant conclusion about the efficacy of the drug, but the study did provide invaluable lessons about: (1) how to quickly set up and run an Ebola trial in close relationship with the community and non-governmental organisations, (2) how to integrate clinical research into healthcare to achieve improved healthcare and (3) the epidemiology of EVD useful for further research (particularly illustrating the frequency and impact of renal dysfunction in the progression of EVD and the prognostic value of low PCR cycle time (Ct) values, suggesting that EVD drug trials should systematically stratify analyses by baseline Ct value as a surrogate of viral load). The JIKI study results also suggest that favipiravir monotherapy merits further study in patients with medium to high viraemia, but not in those with very high viraemia. ${ }^{45}$

In the light of the safety and tolerance profile of favipiravir in humans, favipiravir may have value as a PEP in minimising the progression of disease in any patient and the spread of disease within a population. A recent study of favipiravir for PEP by Jacobs $e t a l^{33}$ in eight probably exposed healthcare workers showed favipiravir's potential to protect from Ebola infection. The healthcare settings in that study are different from those in West Africa, as the healthcare workers were evacuated after the assessment of each exposure level. The results of the risk assessment algorithm employed by Jacobs et al suggest that use of favipiravir for PEP could represent a critically important public health countermeasure to contain infection chains by treating high-risk segments of the populations.

\section{EFFICACIOUS DEPLOYMENT OF AVAILABLE LIFE-SAVING SOLUTIONS \\ Differentiation of therapeutic measures adjusting to local health system constructs}

Determining the optimal EVD treatment regimen for different stages of EVD and different population segments (eg, adults and children) presents several challenges. $^{34} 35$

The preliminary result of the PREVAIL-II randomised clinical trial of Zmapp (Mapp Biopharmaceutical, USA) in Guinea, Liberia and Sierra Leone indicates the potential for monoclonal antibody approaches to treat EVD. ${ }^{36}$ However, given the challenges of inventory management and logistics in regions where the infrastructure for these functions is not well established, orally administered drugs like favipiravir have an advantage over injectable biologics and injectable drugs. Nonetheless, Zmapp and Gilead GS-5734, ${ }^{37} 38$ both of which are administered as injections, may be important for acute infection cases where an orally administered treatment cannot be used. In the light of this need, an intravenous formulation of favipiravir is currently under development.

\section{Strategic multilayered approach}

The study of favipiravir in patients with EVD in Guinea was based on the WHO reaching an ethical consensus about the compassionate use of potential but unregistered medical interventions on satisfaction of certain conditions regarding safety and efficacy. ${ }^{39}$ For the people in the affected countries confronted by EVD and 
similar diseases, however, the issues go far beyond those usually associated with a clinical study. It is essential that all the relevant stakeholders work towards integrating the science of conducting clinical studies of experimental treatments, the practice of medicine to treat the disease, and the life-altering social and economic impact on the communities, nations and regions suffering from an outbreak of EVD. This integration is critical for being able to comprehensively assess and adjust the regime of countermeasures and related healthcare brought to bear on the outbreak in order to maximise local healthcare outcomes. This means that we must combine the knowledge collectively gained from our various experiences to construct and implement healthcare strategies that include tools for reliable prevention, diagnostics and medication. For this purpose, we offer in figure 2 a multilayered approach, based on what we have learnt about EVD care management in Guinea. This approach takes into consideration the critical timing of diagnosis for preventive and therapeutic intervention and the importance of integrating PEP strategy for efficient containment of EVD with an optimum intervention level.

\section{Packaging the diagnosis and care options for locally acceptable field operations}

Compiling a list of the most effective EVD diagnosis and care options in terms of infrastructural and functional elements that will be acceptable at the community level will require community-focused service delivery models that can at the same time introduce and maximise the capacity of currently available technologies and resources and also provide data for analysing the safety
Figure 2 Conceptual diagram of multilayered Ebola virus disease (EVD) case management (based on Guinea experiences if appropriate medical countermeasures available).

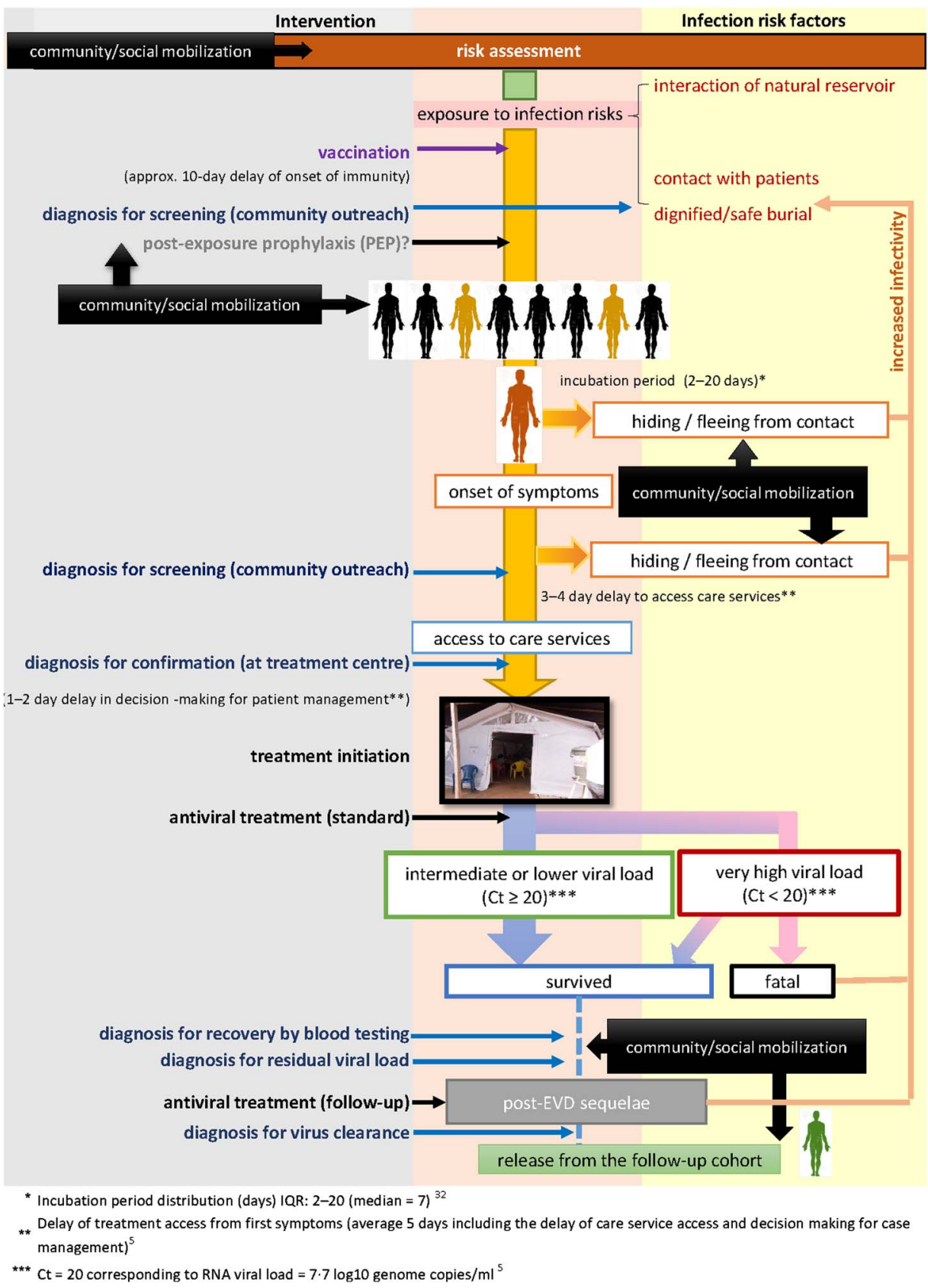


and efficacy of those options and measuring individual and public health outcomes. To establish the most effective countermeasures for EVD, we suggest a reexamination of the adaptability of the currently available primary healthcare approaches employed globally for control of HIV/AIDS, ${ }^{40}$ Malaria and Neglected Tropical Diseases. $^{41}{ }^{42}$ Those approaches usually involve the establishment and deployment of a community-focused, mobile healthcare mechanism ${ }^{43}$ purposefully aimed at strengthening healthcare logistics for contact tracing and adherence to medication. Addressing the unequal impact on men, women and children's health should also be advocated. ${ }^{34} 3544$ Conventional precounselling and postcounselling for testing may be required in a locally acceptable and affordable public healthcare package, as well as providing continued support for health awareness promotion strategies ${ }^{45}$ and reconstructing and integrating health education and nutritional programmes.

\section{CONCLUSIONS}

Lessons learnt by the various researchers, governmental and non-governmental organisations, and clinicians in addressing the 2014-2015 EVD epidemic in West Africa must be collaboratively collected and analysed in a systematic fashion in order to be best prepared at the local level for the next EVD epidemic. In this context, we believe that the field operations with RT-LAMP diagnostic instruments and the clinical study of favipiravir in Guinea provide important information about adapting diagnostic screening and clinical trial procedures in environments characterised by substantial field constraints and allowed trust-building with local communities plagued by limited resources. ${ }^{46}$ Our first-hand knowledge gained from the 2014-2015 EVD outbreak illustrates how collaborative efforts and translational networking in a public health crisis can offer new and productive insights. Through these lessons learnt, we can pragmatically interpret the scientific results of these programmes and collaboratively facilitate discussions among the applicable authorities and members of the global multinational healthcare network for timely and coordinated deployment of resources to achieve the muchneeded public health responses during outbreaks in a coordinated manner. ${ }^{47}$ Furthermore, this type of coordination should ensure a sustainable financial mechanism for being strategically prepared to align and mobilise scientific and $\mathrm{R} \& \mathrm{D}$ pipeline resources at both multilateral and country levels in a systematic manner designed to address the changing epidemiological dynamics of deadly infectious diseases beyond the 20142015 Ebola outbreak. ${ }^{49} 50$

\section{Handling editor Seye Abimbola.}

Acknowledgements The authors express their profound gratitude to Mr Alan $L$ Jakimo, Special Professor of Law of the Hofstra Law School, for his comprehensive editorial contribution to review this article.
Contributors YM conceived and wrote the first draft of the manuscript under TT's overall technical supervision. TS, YK, JY and MT reviewed the specialist topics contained in the manuscript and revised them accordingly in person or by email. DJMM reviewed the subsequent versions and advised on substantive points in the affected countries' context. All authors contributed equally to the planning, writing and revision of this manuscript.

Funding St. Luke's International University, Tokyo, Japan operates as a national coordination hub for international R\&D collaboration under the financial contract of the Japan Agency for Medical Research and Development (AMED) of Japan (grant number:15fk0108039h0001-0003) to conduct the Project on R\&D of Therapeutics and Diagnostics for the Containment of EVD.

Competing interests Toyama Chemicals Co., Ltd. is the developer and supplier of favipiravir for clinical studies and patient care in Guinea as well as for evacuated cases. JY and YK of Nagasaki University are collaborating with Toshiba Medical Systems Corp. to develop an EVD diagnostic kit for use with RT-LAMP methods. Toyama Chemicals Co., Ltd. and Toshiba Medical Systems Corp. are the designees of a project to conduct relevant R\&D activities coordinated by St. Luke's International University, under the AMED contract.

Provenance and peer review Not commissioned; externally peer reviewed.

Data sharing statement No additional data are available.

Open Access This is an Open Access article distributed in accordance with the Creative Commons Attribution Non Commercial (CC BY-NC 4.0) license, which permits others to distribute, remix, adapt, build upon this work noncommercially, and license their derivative works on different terms, provided the original work is properly cited and the use is non-commercial. See: http:// creativecommons.org/licenses/by-nc/4.0/

\section{REFERENCES}

1. Faye O, Boëlle PY, Heleze E, et al. Chains of transmission and control of Ebola virus disease in Conakry, Guinea, in 2014: an observational study. Lancet Infect Dis 2015;15:320-6.

2. Mohammadi D. First trials for Ebola treatments announced. Lancet 2014;384:1833.

3. Mendoza EJ, Qiu X, Kobinger GP. Progression of Ebola therapeutics during the 2014-2015 outbreak. Trends Mol Med 2016;22:164-73.

4. Sissoko D, Folkesson E, Abdoul M, et al. Favipiravir in patients with Ebola virus disease: early results of the JIKI trial in Guinea. Abstract No. 103-ALB. Washington, USA: Presented at the CROI, 2015.

5. Sissoko D, Laouenan C, Folkesson E, et al., JIKI Study Group. Experimental treatment with favipiravir for Ebola virus disease (the JIKI Trial): a historically-controlled, single arm proof-of-concept trial in Guinea. PLoS Med 2016;13:e1001967.

6. Mentré F, Taburet AM, Guedj J, et al. Dose regimen of favipiravir for Ebola virus disease. Lancet Infect Dis 2015;15:150-1.

7. World Health Organization. Ebola Response Phase 3. Framework for achieving and sustaining a resilient zero. Geneva, Switzerland: WHO, September 2015.

8. Fischer RJ, Judson S, Miazgowicz K, et al. Ebola virus persistence in semen ex vivo. Emerg Infect Dis 2016;22:289-91.

9. Thorson A, Formenty P, Lofthouse C, et al. Systematic review of the literature on viral persistence and sexual transmission from recovered Ebola survivors: evidence and recommendations. BMJ 2016;6:e008859.

10. Deen GF, Knust B, Broutet N, et al. Ebola RNA persistence in semen of Ebola virus disease survivors-preliminary report. $N$ Eng J Med 2015. doi:10.1056/NEJMoa1511410

11. Notomi T, Okayama $\mathrm{H}$, Masubuchi $\mathrm{H}$, et al. Loop-mediated isothermal amplification of DNA. Nucleic Acids Res 2000;28:E63.

12. Furuta Y, Gowen BB, Takahashi K, et al. Favipiravir (T-705), a novel viral RNA polymerase inhibitor. Antiviral Res 2013;100:446-54.

13. Furuta $Y$, Takahashi $K$, Fukuda $Y$, et al. In vitro and in vivo activities of anti-influenza virus compound T-705. Antimicrob Agents Chemother 2002;46:977-81.

14. World Health Organization. Annex 3: Considerations for the selection of rapid antigen detection tests for the diagnosis of Ebola virus disease. Emergency Guidance. Selection and Use of Ebola in vitro diagnostic (IVD) assays. Geneva, Switzerland: WHO, June 2015. 
15. Walker NF, Brown CS, Youkee D, et al. Evaluation of a point-of-care blood test for identification of Ebola virus disease at Ebola holding unites, Western Area, Sierra Leone, January to February 2015. Euro Surveill 2015;20:pii: 21073.

16. Leroy EM, Baize S, Lu CY, et al. Diagnosis of Ebola Haemorrhagic Fever by RT-PCR in an Epidemic Setting. J Med Virol 2000;60:463-7.

17. Broadhurst MJ, Kelly JD, Miller A, et al. ReEBOV Antigen Rapid Test kit for point-of-care and laboratory-based testing for Ebola virus disease: a field validation study. Lancet 2015;386;867-74.

18. Boisen ML, Cross RW, Hartnett JN, et al. Field validation of the ReEBOV Antigen Rapid Test for point-of-care diagnosis of Ebola virus infection. J Infect Dis 2016;214 (Suppl 3):S203-9.

19. ReEBOV Antigen Rapid Test (Ebolavirus VP40 Antigen Detection), Instruction for Use. For Use under Emergency Use Authorization (EUA) Only (in vitro diagnostic use), Corgenix, Inc. Colorado, USA. 2015.

20. Rieger T, Kerber R, El Halas H, et al. Evaluation of RealStar reverse transcription-polymerase chain reaction kits for filovirus detection in the laboratory and field. J Infect Dis 2016;214(Suppl 3):S243-9.

21. Cherpillod $\mathrm{P}$, Schibler M, Vieille $\mathrm{G}$, et al. Ebola virus disease diagnosis by real-time RT-PCR: a comparative study of 11 different procedures. J Clin Virol 2016;77:9-14.

22. Janvier F, Gorbatch S, Queval L, et al. Difficulties of interpretation of Zaire Ebola Virus PCR results and implication in the field. $J$ Clin Virol 2015;67:36-7.

23. Panning $\mathrm{M}$, Laue $\mathrm{T}$, Olsjlager $\mathrm{S}$, et al. Diagnostic reversetranscription polymerase chain reaction kit for filovirus based on the strain collections of all European biosafety level 4 laboratories. $J$ Infect Dis 2007;196(Suppl 2):S199-204.

24. Jansen van Vuren P, Grobbelaar A, Storm N, et al. Comparative evaluation of the Diagnostic Performance of the Prototype Cepheid GeneXpert Ebola Assay. J Clin Microbiol 2016;54:359-67.

25. Pinsky BA, Shoo MK, Sandlund J, et al. Analytical performance characteristics of the Cepheid GeneXpert Ebola assay for the detection of Ebola virus. PLOS ONE 2015;10:e0145896.

26. Kurosaki Y, Magassouba N, Oloniniyi OK, et al. Development and evaluation of reverse transcription loop-mediated isothermal amplification (RT-LAMP) assay coupled with a portable device for rapid diagnosis of Ebola virus disease in Guinea. PLoS Negl Trop Dis 2016;10:e0004472.

27. Nouvellet $\mathrm{P}$, Garske T, Mills HL, et al. The role of rapid diagnostics in managing Ebola epidemics. Nature 2015;528:S109-116.

28. Faye O, Faye O, Soropogui B, et al. Development and deployment of a rapid recombinase polymerase amplification Ebola virus detection assay in Guinea in 2015. Euro Surveill 2015;20:20(44). doi:10.2807/1560-7917.ES.2015.20.44.30053

29. Henao-Restrepo AM, Longini Intramuscular, Egger M, et al. Efficacy and effectiveness of an rVSV-vectored vaccine expressing Ebola surface glycoprotein: interim results from the Guinea ring vaccination cluster-randomized trial. Lancet 2015;386;857-86.

30. Sridhar S. Clinical development of Ebola vaccines. Ther Adv Vaccines 2015:3:125-38.

31. High Council for Public Health. Opinion. Relating to the management of healthcare worker $(\mathrm{HCW})$ in care settings who are victims of an AEB/AEV from a patient who is a confirmed index case of Ebola virus disease. Haut Conseil de la Santé Publique, France. 4 December 2014. http://www.hcsp.fr/Explore.cgi/ avisrapportsdomaine?clefr=471 (accessed 5 Sep 2016).

32. Van Kerkhove MD, Bento Al, Mills HL, et al. A review of epidemiological parameters from Ebola outbreaks to inform early public health decision-making. Sci Data 2015;2:150019 http://dx.doi. org/10.1038/sdata.2015.19.

33. Jacobs M, Aarons E, Bhagani S, et al. Post-exposure prophylaxis against Ebola disease with experimental antiviral agents: a case-series of healthcare workers. Lancet Inf Dis 2015;15; $1300-4$.

34. Menéndez C, Lucas A, Munguambe $\mathrm{K}$, et al. Ebola crisis: the unequal impact on women and children's health. Lancet 2015;3: e130.

35. Baggi FM, Taybi A, Kurth A, et al. Management of pregnant women infected with Ebola virus in a treatment centre in Guinea, June 2014. Euro Surveill 2014;19:pii: 20983

36. Davey RT. PREVAIL II: A Randomized Controlled Trial of ZMapp TM in Acute Ebola Virus Infection. Presented at the CRO 2016, Abstract No. 77LB. Boston, MA, USA. http://www. croiconference.org/sessions/prevail-ii-randomized-controlledtrial-zmapp\%E2\%84\%A2-acute-ebola-virus-infection (accessed 5 Sep 2016).

37. Warren T, Jordan R, Lo M, et al. Nucleotide Prodrug GS-5734 is a broad-spectrum filovirus inhibitor that provides complete therapeutic protection against the development of Ebola virus disease (EVD) in infected non-human primates. Open Forum Infect Dis (Fall 2015) 2 (suppl 1): doi: 10.1093/ofid/ofv130.02.

38. Gilead Sciences, . Gilead Provides Update on Investigational Compound, GS-5734, for the Treatment of Ebola Virus Disease. 21 October 2015. http://www.businesswire.com/news/home/ 20151021005958/en/ (accessed 5 Sep 2016).

39. World Health Organization (14 August 2014). Ethical considerations for use of unregistered interventions for Ebola virus disease (EVD). http://www.who.int/mediacentre/news/statements/2014/ebola-ethicalreview-summary/en/ (accessed 5 Sep 2016).

40. Piot P, Quinn TC. Response to the AIDS pandemic - a global health model. N Engl J Med 2013;368:2210-18.

41. Bardosh K. Global aspirations, local realities: the role of social science research in controlling neglected tropical diseases. Infect Dis Poverty 2014;3:35.

42. Aagaard-Hansen J, Chaignat CL. Neglected tropical diseases: equity and social determinants. In: Blas E, Kurup AS, eds. Equity, social determinants and public health programmes. Geneva, Switzerland: WHO, 2010:135-57.

43. Stamm LV. Ebola virus disease: rapid diagnosis and timely case reporting are critical to the early response for outbreak control. Am Soc Trop Med Hyg 2015;93:438-40.

44. Bouazza N, Treluyer JM, Foissac F, et al. Favipiravir for children with Ebola. Lancet 2015;385:603-4.

45. World Health Organization. Interim guidance. Clinical care for survivors of ebola virus disease. Geneva, Switzerland: WHO. 25 January 2016.

46. Farrar JJ, Piot P. The Ebola emergency-immediate action, ongoing strategy. N Eng J Med 2014;371:1545-6.

47. Gates B. The next epidemic-lessons from Ebola. N Eng J Med 2015;372:1381-4

48. Gostin LO, Friedman EA. A retrospective and prospective analysis of the West African Ebola virus disease epidemic: robust national health systems at the foundation and an empowered $\mathrm{WHO}$ at the apex. Lancet 2015;385:1902-9.

49. Butler $D$. The next time. The world is ill-prepared for the next epidemic or pandemic. But the horror of the Ebola outbreak in West Africa may drive change. Nature 2015;524:22-5.

50. Dzau VJ, Sands P. Beyond the Ebola Battle- winning the war against future epidemics N Engl J Med 2016;375:203-4. 